\title{
Cerro Borreguero. Un yacimiento clave para estudiar la transición entre el Bronce Final y el periodo tartésico en el valle del Guadiana*
}

\author{
Cerro Borreguero. An archaeological site for the transition from the Final Bronze Age \\ to the Tartessian period in the Guadiana Valley
}

Sebastián Celestino Pérez ${ }^{\mathrm{a}}$ y Esther Rodríguez González ${ }^{\mathrm{a}}$

\section{RESUMEN}

Se recogen en el presente trabajo los resultados obtenidos tras la ejecución de cinco campañas de excavación en el yacimiento de Cerro Borreguero (Zalamea de la Serena, Badajoz). Estos trabajos han permitido documentar la existencia de tres momentos de ocupación que se extienden entre el siglo IX ane y I ane con un hiatus pronunciado entre las etapas protohistórica y romana. La aparición de una cabaña oval en convivencia con una construcción de planta rectangular nos sitúa ante el primer yacimiento de transición entre el Bronce Final y la I Edad del Hierro en el valle medio del Guadiana.

\begin{abstract}
This work summarizes the results of five excavation campaigns undertaken at the site of Cerro Borreguero (Zalamea de la Serena, Badajoz). Our work shows the existence of three moments of occupation between the ninth and the first century BCE, with a noticeable hiatus between the Protohistoric and Roman periods. The appearance of an oval hut along with a rectangular construction indicates that this is the first archaeological site in the middle Guadiana valley that dates to the transition between the Late Bronze Age and the Early Iron Age.
\end{abstract}

Palabras clave: Valle medio del Guadiana; Transición; Arquitectura; Cabaña oval.

Key words: Middle Valley of the Guadiana River; Transition; Architecture; Oval hut.

\section{INTRODUCCIÓN}

El yacimiento arqueológico de Cerro Borreguero se localiza al noroeste del municipio de Zalamea de la Serena (Badajoz), a tan solo 2,5 km al oeste del enclave de Cancho Roano. (Fig. 1)

Se trata de una elevación artificial sobre un afloramiento de cuarcitas, situada a menos de $100 \mathrm{~m}$ de la margen derecha del río Ortigas, cuyo curso desemboca en el Guadiana $30 \mathrm{~km}$ más arriba, muy próximo a la necrópolis tartésica de Medellín.

Aunque en origen se trataba de un asentamiento en llano, el abandono paulatino y la reforma de los diferentes edificios de época protohistórica han provocado que en la actualidad se conforme como un montículo artificial de tendencia ovalada y orientación este-oeste que despunta en un paisaje de dehesa. No obstante, su desnivel es muy desigual debido a que no todas las caras de la elevación presentan igual estado de conservación; así, mientras su lado sur se encuentra al mismo nivel que el suelo natural, su cara norte es muy pronunciada y, por lo tanto, es donde mejor se conserva tanto el alzado del túmulo como las construcciones que se ocultan debajo. Esta desigualdad se debe a que el yacimiento se localizada dentro de una propiedad destinada al cultivo de secano, por lo que durante las últimas décadas del siglo XX fue víctima de las labores agrícolas que se desarrollaron en su entorno y que, como veremos a continuación, son la causa de parte de la destrucción del enclave. Sin embargo, la alteración que han sufrido las edificaciones protohistóricas no se

* Este trabajo se inserta dentro del proyecto del Plan Estatal de Investigación I+D “Construyendo Tarteso. Análisis constructivo, espacial y territorial de un modelo arquitectónico en el valle medio del Guadiana" (HAR2015-63788-P).

${ }^{a}$ Instituto de Arqueología-Mérida, CSIC-Junta de Extremadura. Plaza de España, 15. 06800-Mérida. España. Correos e.: scelestino@iam.csic.es https://orcid.org/0000-0002-2050-7090; esther.rodrigez@iam.csic.es https://orcid.org/0000-0002-5813-9035 


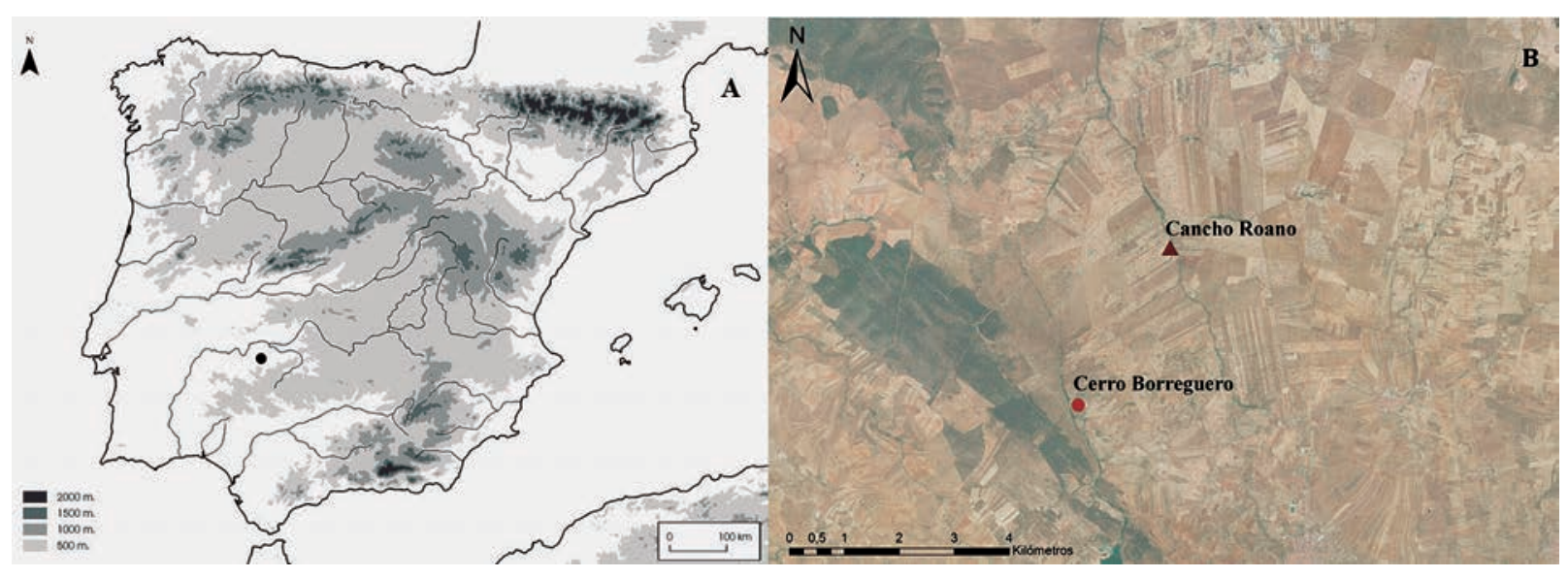

Fig. 1. Localización del yacimiento de Cerro Borreguero (Zalamea de la Serena, Badajoz) en la Península Ibérica (A) y en la comarca de La Serena (B) (obra derivada de PNOA2016 CC-BY ign.es, figura en color en la edición electrónica).

deben únicamente a los trabajos del campo, sino a la existencia de una construcción romana $\mathrm{y}$, en concreto, a sus potentes cimientos, que han actuado con cierta agresividad sobre las fases más antiguas. Este edificio de época romana tiene un gran interés, pero será objeto de estudio en otro lugar para así centrarnos ahora en las fases arqueológicas que aquí queremos tratar.

El yacimiento de Cerro Borreguero ha sido objeto de cinco campañas de excavación que han permitido documentar una completa secuencia de ocupación que arranca en la Prehistoria Reciente y se prolonga hasta época imperial, con la presencia de un hiatus entre los siglos VI ane y I ane. El interés del presente trabajo se ciñe a la presentación y análisis de los edificios fechados entre el Bronce Final y la I Edad del Hierro al constituir, por un lado, el único ejemplo de arquitectura del Bronce Final documentada en el valle medio del Guadiana y, por el otro, el único yacimiento a partir del cual se puede realizar un análisis constructivo de la evolución de la técnica edilicia entre el Bronce Final y la I Edad del Hierro dentro de este espacio geográfico.

\section{FASES DE OCUPACIÓN}

Los resultados derivados de los análisis constructivos llevados a cabo en Cerro Borreguero han permitido distinguir tres fases de ocupación: la fase I o etapa romana que comenzaría en época republicana y pervivió hasta época imperial a tenor de las cerámicas de paredes finas que aparecen en los niveles superficiales; la fase II o etapa de la I Edad del Hierro, que cuenta a su vez con dos subfases constructivas que caracterizaremos más adelante $\mathrm{y}$, por último, la fase III que se adscribe al Bronce Final a partir de la existencia de una cabaña oval que hemos podido datar en el siglo IX ane a partir de análisis de C14.
La Fase I se corresponde así con la época imperial romana y será objeto de un trabajo pormenorizado en otro foro por su interés e imbricación con el paisaje de recintos-torres de esta época que domina la comarca de La Serena (Mayoral et al. 2011, con bibliografía; Celestino y Cazorla 2013), por lo que en este estudio solo serán objeto de análisis las fases correspondientes con el Bronce Final y la I Edad del Hierro.

\subsection{Fase II: Hierro I (siglos VIII-VI ane)}

La ocupación protohistórica de Cerro Borreguero está claramente diferenciada en dos subfases. La primera, la fase IIa, se corresponde con el último momento de ocupación del enclave en la I Edad del Hierro hacia principios del siglo VI ane, momento en el que también se fecha la amortización de todas sus estancias mediante el relleno con piedras de granito irregulares y sin trabar para, posteriormente, sellar todo el espacio con una gran capa de arcilla roja que confiere a la elevación su apariencia tumular. Esta práctica no es exclusiva del yacimiento de Cerro Borreguero, pues una capa de arcilla roja sellaba también el túmulo que ocultaba la construcción de Cancho Roano.

Al igual que el edificio de cronología romana, la planta conservada en esta fase IIb también tiene forma de ' $\mathrm{L}$ ' invertida, o al menos así lo muestran los restos que han podido ser excavados (Fig. 2). Sin embargo, no se puede descartar la posibilidad de que las labores agrícolas hayan arrasado parte de la construcción en su extremo sur y que, en origen, el edificio de la fase IIa tuviera una planta similar a la que poseen edificios más modernos como Cancho Roano "A" o La Mata, es decir, de tendencia cuadrangular. No obstante, y aunque no existen muchos paralelos de 


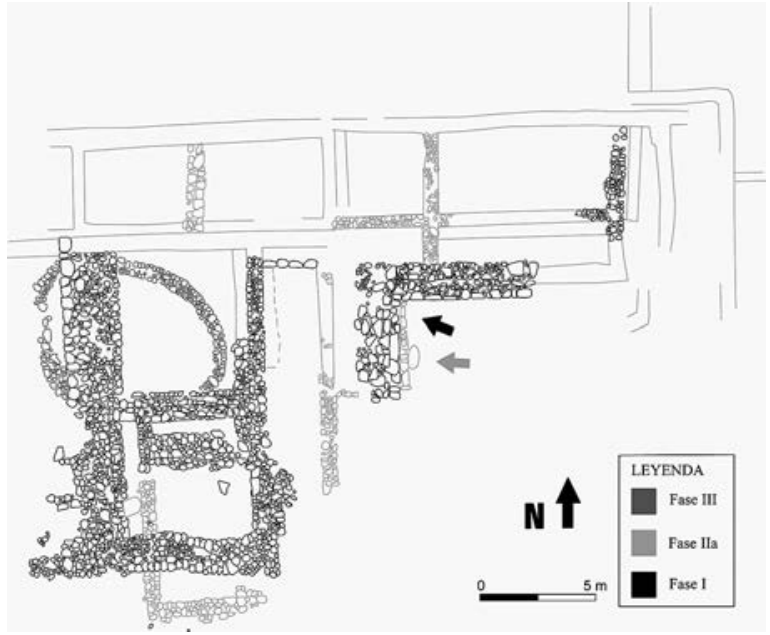

Fig. 2. Planimetría del edificio correspondiente a la fase IIa de Cerro Borreguero (Zalamea de la Serena, Badajoz).

edificios en 'L' dentro de la Protohistoria del suroeste peninsular, lo cierto es que esta posible planta en ' $\mathrm{L}$ ' guarda similitudes con la última fase constructiva del edificio MN23 de Castro dos Ratinhos (Berrocal y Silva 2010), cuando al edificio de planta rectangular se le adosa una nueva estancia, U.A. M2, que le da a la construcción una aparente forma en ' $\mathrm{L}$ ' invertida (Prados 2010: 268) (Fig. 3). Pero a pesar de las similitudes que puedan existir entre las construcciones de ambos asentamientos, cuyo detalle más destacado es la presencia de una convivencia entre edificios de planta oval y rectangular, considerar la planta en ' $\mathrm{L}$ ' de Cerro Borreguero como la planta original del último edificio protohistórico genera algunas dudas que se fundamentan, principalmente, en que la estancia que marca el eje más corto, norte-sur, es la habitación de mayor tamaño dentro de la construcción (estancia 100), además de ser la que presenta mayores particularidades dentro de esta última fase constructiva.

La estancia 100 del edificio protohistórico de Cerro Borreguero (Fig. 4) tiene planta ligeramente rectangular, de $5 \times 6 \mathrm{~m}$, aproximadamente, pues solo conocemos los cimientos de tres de sus muros al estar soterrado el occidental bajo la terraza romana; así mismo, el hecho de que parte de la construcción romana se levantara sobre los muros protohistóricos nos impide conocer la ubicación del vano de acceso. Los cimientos conservan hasta $1 \mathrm{~m}$ de alzado en algunos de sus puntos y fueron construidos con cuarcitas de mediano y pequeño tamaño trabadas con barro sobre las que se levantaron muros de adobe, de entre $0,45-0,55 \mathrm{~m}$, que fueron documentados en el relleno de la habitación durante su excavación. Por último, el suelo de la estancia, como el resto de los suelos de las habitaciones de esta fase constructiva, se realizó con arcilla roja apisonada dispuesta sobre una lechada de arcilla marrón que da una gran consistencia a los pavimentos.

La singularidad de esta habitación 100, además de por ser la que mayores dimensiones posee, radica en la presencia de un hogar circular centrado en la mitad meridional de la estancia. La estructura está realizada con arcilla sobre una base de piedras. En su extremo septentrional se dispusieron varios ladrillos de adobe en posición horizontal que debieron servir para contener las llamas. Fruto de su uso la plataforma central o focus conservaba aún la cama de cenizas y arcilla refractada por la acción del fuego (Fig. 5). La excavación de la estructura permitió documentar varios niveles de uso diferenciados por la presencia de sucesivas capas de arcillas de distinto color y textura. Del interior de la estructura se recuperó un interesante lote de cerámicas fabricadas a mano y decoradas, en su gran mayoría, con escobillados. Finalmente, el hogar apoya sobre un relleno de piedras de granito de mediano y gran tamaño que sirvieron para colmatar la construcción de la fase III de Cerro Borreguero, lo que demuestra que la estructura circular ya existía cuando la construcción de la fase III estaba en funcionamiento.

El segundo elemento que destaca sobre el pavimento rojo de esta habitación es la presencia de una banda curva de unos $12 \mathrm{~cm}$ de anchura que atraviesa todo el

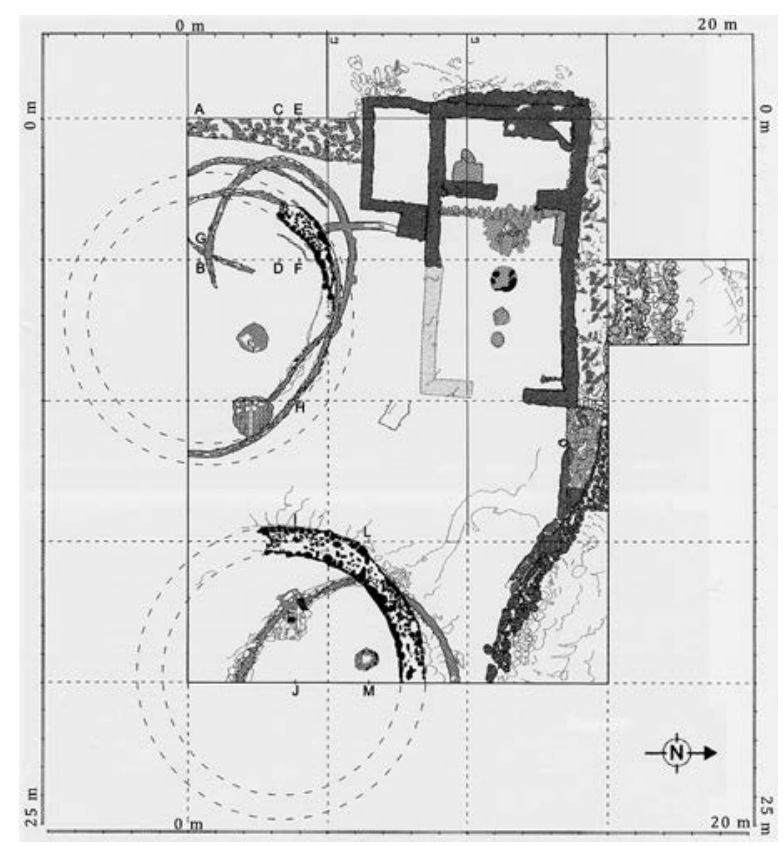

Fig. 3. Planta de los edificios hallados en las excavaciones de Castro dos Ratinhos (a partir de Berrocal y Silva 2010: 248) 


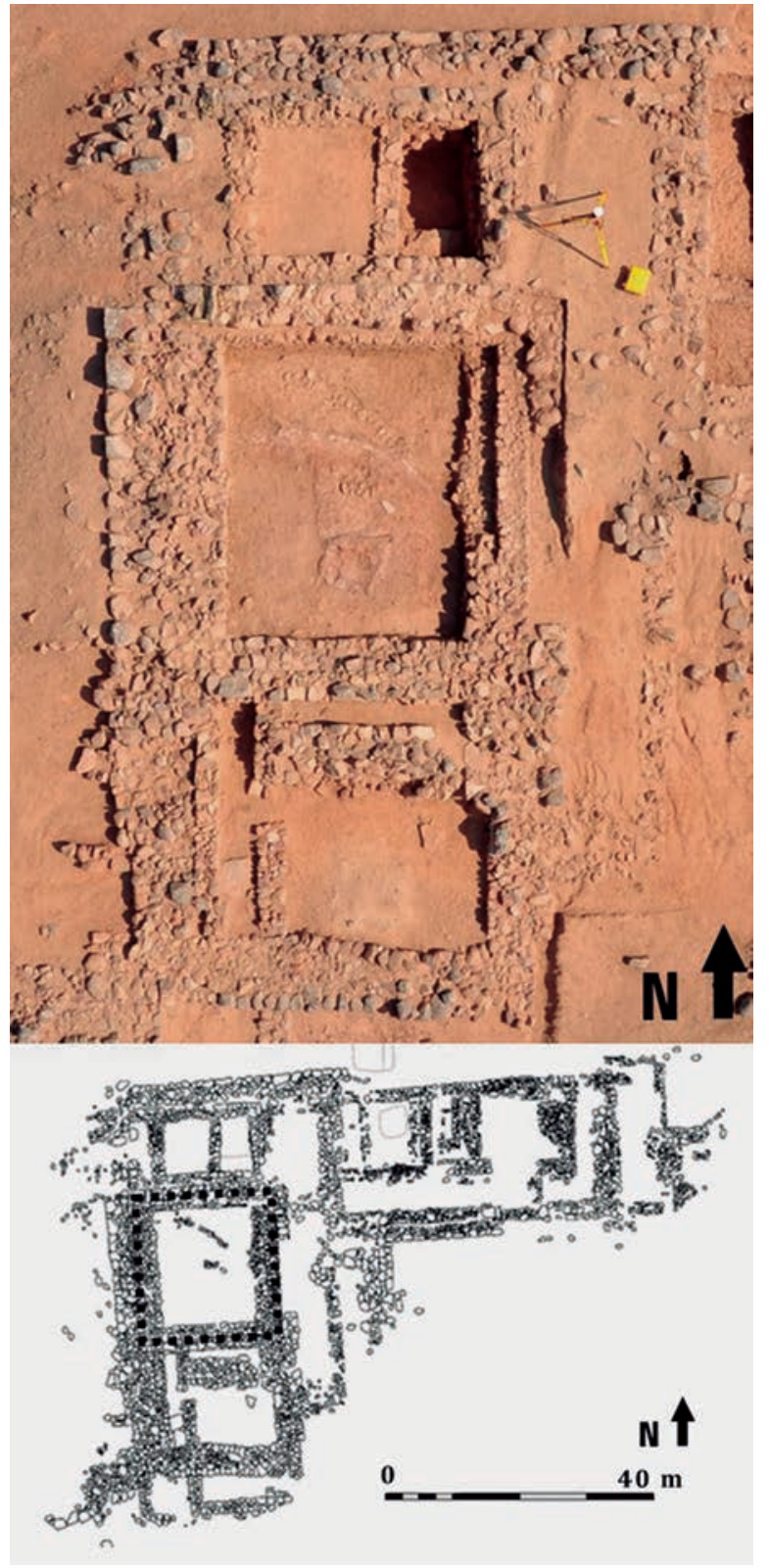

Fig. 4. Localización en el plano general de Cerro Borreguero (Zalamea de la Serena, Badajoz) de la estancia 100 en la Fase IIa y vista aérea de la misma. Fotografía: Proyecto de Investigación "Construyendo Tarteso”, HAR 2015-63788-P (en color en la edición electrónica).

espacio en dirección oeste-este (Fig. 6), perdiéndose bajo los límites de la estancia y de las terrazas romanas. Dicha banda está realizada con adobes de unos $6 \mathrm{~cm}$ de potencia insertos en el pavimento y pintados de blanco. $\mathrm{Su}$ presencia fue interpretada como los restos de un elemento simbólico del espacio cuyo significado resultaba en un primer momento desconocido. Solo cuando se procedió a excavar el suelo rojo de la estancia se pudo comprobar que la banda blanca estaba señalando el trazado de una gran cabaña ovalada que se había amortizado para construir el último edificio protohistórico.

En cuanto a la cubierta de este amplio espacio carecemos de documentación básica para interpretarla. Cabe suponer que la habitación 100, así como el resto del edificio, contaría con techos planos realizados con traviesas de madera que posteriormente se cubrirían por ramaje y barro como es habitual en las construcciones de la época; sin embargo, y a pesar de la gran superficie de la estancia, no se detectaron agujeros de poste, restos de vigas de madera o improntas de pilares de adobe, por lo que no podemos descartar que la habitación hubiera estado al descubierto como parece

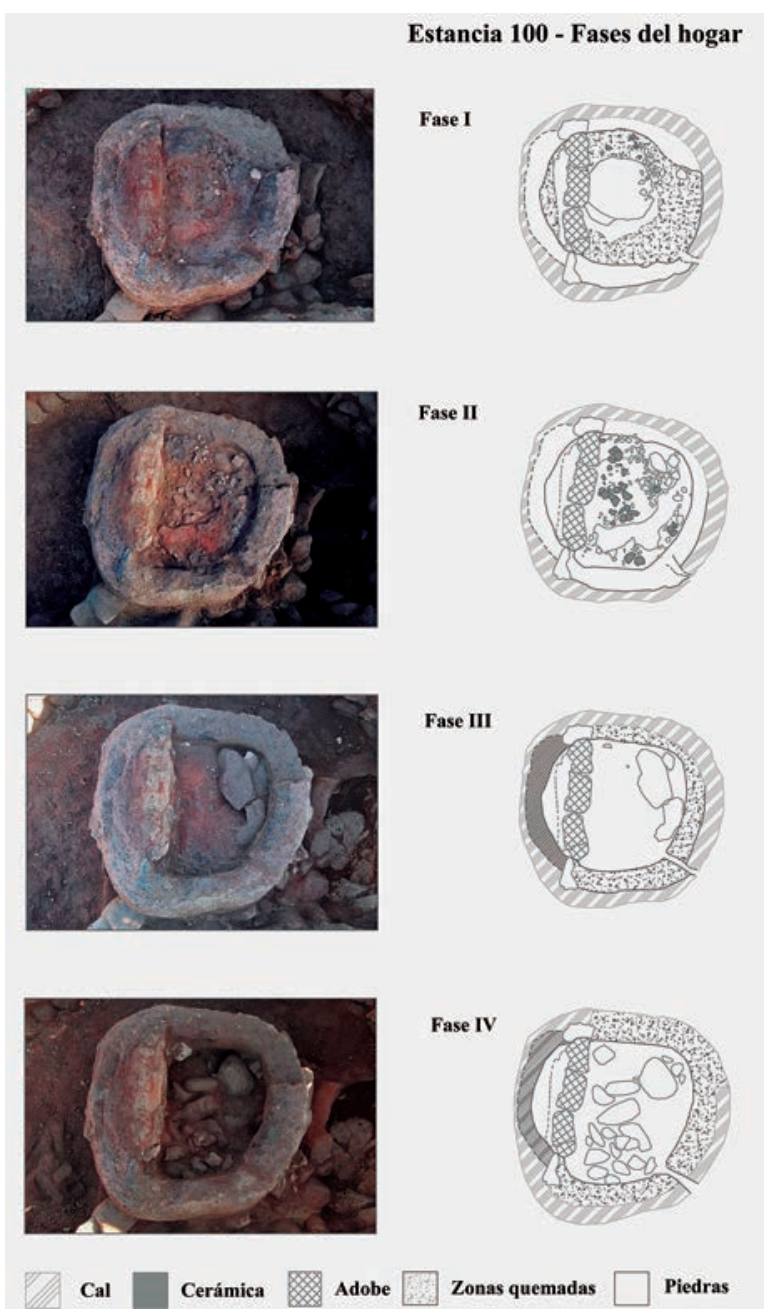

Fig. 5. Secuencia de excavación del hogar de la estancia 100 de Cerro Borreguero (Zalamea de la Serena, Badajoz) (en color en la edición electrónica). 


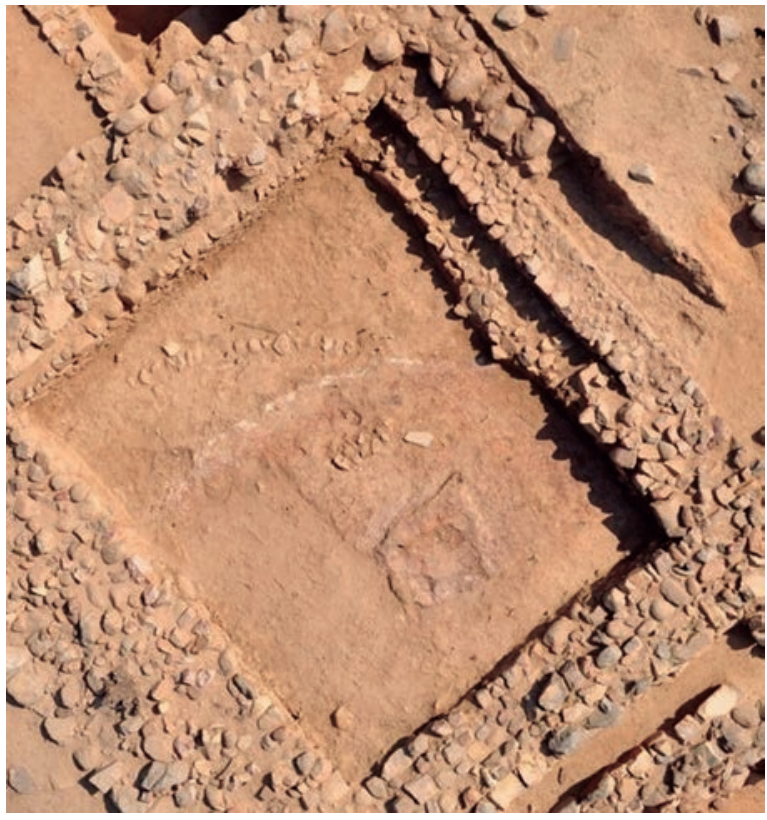

Fig. 6. Vista aérea de la estancia 100 de Cerro Borreguero (Zalamea de la Serena, Badajoz) en la Fase IIa con el detalle de la banda blanca dibujada sobre el pavimento de la misma. Fotografía: Proyecto de Investigación "Construyendo Tarteso", HAR 2015-63788-P (en color en la edición electrónica).

indicar la leve inclinación del pavimento, aunque no se han encontrado canalizaciones para la evacuación del agua que, por otro parte, podrían estar soterradas bajo la terraza romana. Por último, la estancia contaba con un banco corrido adosado al muro este, hoy prácticamente desaparecido, pues solo se conserva el arranque de sus cimientos. La estancia 100, junto al resto del edificio correspondiente a la fase IIa, se puede fechar entre finales del siglo VII e inicios del siglo VI ane a tenor de los materiales cerámicos documentados.

Lamentablemente, el edificio romano impide conocer buena parte de la planta general del edificio protohistórico, incluso nos ha imposibilitado individualizar algunos de los espacios que se corresponderían con las diferentes estancias que conformarían la construcción. Así, por ejemplo, la escalinata por la que se accede al edificio romano impide analizar cómo era la comunicación entre la estancia 100 y los espacios adyacentes. Hasta el momento, y a partir de los cimientos conservados, hemos podido individualizar hasta ocho espacios, de los cuales seis parecen corresponderse con estancias y los dos restantes con pasillos de distribución que comunicarían el edificio en sentido este-oeste y norte-sur. En cuanto a los elementos arquitectónicos secundarios, sabemos que algunas de estas estancias contaban con pequeños hogares encargados de calentar e iluminar los habitáculos. Este es el caso de una de las estancias de la zona 600, donde pudo documentarse la existencia de un hogar construido a base de pequeños cantos de río en cuyo contorno la arcilla se encontraba completamente refractada y mezclada con cenizas. El acceso a este edificio de la Fase IIa se practicaría por el este, en sintonía con los monumentos más destacados que conocemos de época tartésica, tanto del Guadiana, caso de Cancho Roano o La Mata, como del Guadalquivir, caso del Carambolo o Coria del Río (Esteban y Escacena 2013), estos últimos de adscripción fenicia según las más recientes investigaciones (Fernández Flores y Rodríguez Azogue 2007: 269; Escacena 2011). No obstante, y desde nuestro punto de vista, si bien no albergamos dudas sobre el origen fenicio de los primigenios santuarios del Guadalquivir, parece obvio que a partir del siglo VIII ane, cuando precisamente se desarrollan los edificios mejor conocidos de Caura y el Carambolo, ya podemos hablar de santuarios tartésicos, pues es cuando los componentes fenicio e indígena interactúan y se desarrolla lo que conocemos como cultura tartésica (Celestino y López-Ruiz 2016: 232). Por último, frente a esta entrada oriental se extendía un gran patio abierto pavimentado de arcilla roja que ocuparía una posición central en el que caso de que el edificio contara con una disposición simétrica de esquema en ' $U$ '.

Otra de las características que llaman la atención en el yacimiento de Cerro Borreguero, y que se acentúa en la fase IIa, es que todas las estancias documentadas aparecen prácticamente vacías, colmatadas con cantos de río de mediano tamaño en seco que posteriormente fueron cubiertos con la capa de arcilla roja que sella esta última fase constructiva $\mathrm{y}$, por ende, la amortización del edificio. La escasez de materiales arqueológicos, el hecho de que todas las estancias estuvieran colmatadas siguiendo el mismo proceso y la ausencia de niveles de incendio en la parte superior del túmulo, como ocurre en otras construcciones posteriores del Guadiana Medio, caso de Cancho Roano o el túmulo de "Casas del Turuñuelo" (Rodríguez González y Celestino, 2017), indica que el abandono debió ser intencionado y no traumático. Por último, subrayar la escasez de materiales al tratarse de un nivel de abandono, pero destacan las cerámicas realizadas a mano, mientras que las elaboradas a torno son testimoniales.

La fase IIb es sin duda la que presenta mayor interés arquitectónico y arqueológico; sin embargo, es la que nos ha llegado más desdibujada por la superposición de muros correspondientes a la fase inmediatamente anterior y a la construcción de las terrazas romanas, lo que dificulta el conocimiento de esta etapa. De esta fase solo se ha documentado alguno de sus paramentos y un único pavimento, insuficientes en todo caso para presentar una planta completa de la edificación de la que forman parte. Sin embargo, la particularidad de esta fase, fechada entre los siglos VIII-VII ane, reside en la relación directa que 
existe entre este primer edificio de muros rectos y la cabaña oval que caracteriza a la fase III.

El edificio de la fase IIb se localiza en el sector más septentrional del yacimiento, sobre el suelo natural, lo que significa que, a diferencia de las construcciones de la fase IIa, el edificio de esta etapa se levantó en el llano; es decir, a los pies de la cabaña oval, construida sobre la cota más alta del terreno. Aparentemente, por los paramentos que hemos podido individualizar, parece tratarse de un edificio rectangular con eje este-oeste de más de $21 \mathrm{~m}$ de largo. La excavación de algunos de los espacios individualizados del edificio de la fase IIa nos ha permitido comprobar que el interior de este primer edificio cuadrangular también estaba compartimentado.

Los escasos muros documentados de esta fase IIb conservan parte de su alzado de adobe e incluso restos del encalado que los enlució, lo que parece demostrar que la construcción fue amortizada para levantar el edificio posterior de la fase IIa, de mayor dimensión, pero sin destruir por completo el anterior, sino solo variando la disposición de su planta. De esta forma, solo los tramos afectados por la construcción de los nuevos cimientos se han visto alterados. Sin embargo, lo más relevante de esta fase constructiva es el diseño de algunas de sus esquinas. En efecto, tras la excavación del extremo noreste de la elevación, se pudo comprobar que sus esquinas son redondeadas, es decir, que no traban en ángulo recto (Fig. 7). Esta circunstancia se debe, probablemente, a la falta de pericia para levantar muros completamente rectos, lo que demostraría que estamos ante un ensayo arquitectónico que no culminará con éxito hasta la fase posterior.

Esta circunstancia, sin duda singular en el ámbito geográfico donde se desarrolla este estudio, lleva a plantearse cuestiones tan interesantes como la autoría de quieres ejecutaron la obra. En este sentido, contamos de nuevo con el ejemplo documentado en el edificio MN23 de Castro dos Ratinhos, donde los autores vieron la clara presencia de un arquitecto fenicio o procedente del área tartésica en función del patrón de medida empleado para levantar el edificio (Berrocal et al. 2012: 177), si bien las carencias detectadas en la ejecución de la obra las atribuyen al empleo de mano de obra indígena (Prados 2010: 267). Sin embargo, el ejemplo hallado en Cerro Borreguero parece que más bien correspondería con un proyecto planteado y ejecutado por alguien que ha visto o conoce la existencia de edificios de planta cuadrangular pero que, sin embargo, desconoce la técnica completa para llevar a cabo su construcción, de ahí que el resultado sea un edificio de muros rectos pero con esquinas redondeadas. Así mismo, la ausencia de materiales de importación y el anecdótico porcentaje de cerámicas elaboradas a torno parece avalar que la autoría de la obra debió quedar en manos indígenas.

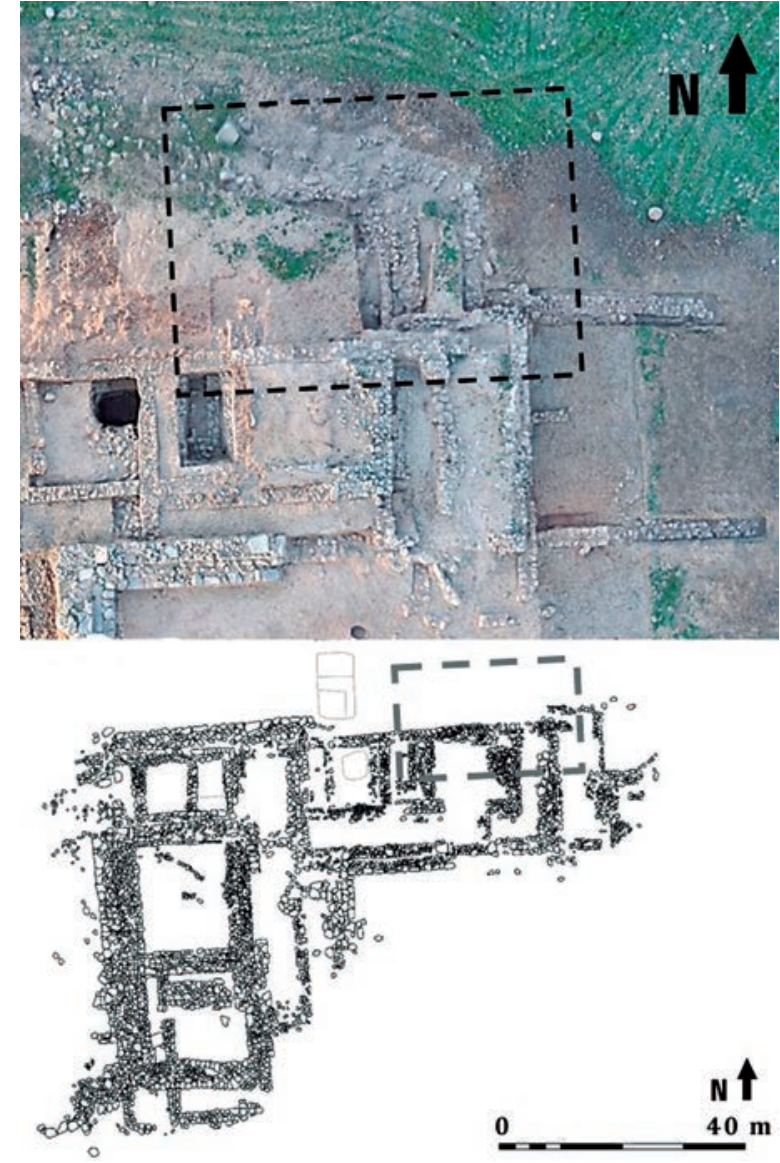

Fig. 7. Detalle del paramento de cierre del edificio de la Fase IIb en la esquina noreste de Cerro Borreguero (Zalamea de la Serena, Badajoz). Fotografía: Proyecto de Investigación "Construyendo Tarteso", HAR 2015-63788-P (en color en la edición electrónica).

\subsection{Fase III. Bronce Final (siglo IX ane)}

La fase III de Cerro Borreguero se corresponde con la primera ocupación del enclave. Está caracterizada por la presencia de una estructura ovalada de grandes dimensiones ubicada en la parte más elevada del paisaje. La cabaña, con una orientación oeste-este, conserva casi todo su trazado oriental, coincidente con la franja blanca descrita en la fase IIa que se detectó sobre el pavimento rojo de la estancia 100. El resto de los cimientos de la cabaña están ocultos bajo las potentes terrazas de época romana, si bien se ha podido delimitar su extremo occidental al asomar ligeramente bajo la terraza romana que cierra el edificio por ese mismo lado (Fig. 8).

La cabaña apoya sobre la roca natural y conserva la totalidad del alzado de sus cimientos, compuestos por tres hiladas de piedras que alcanzan los $60 \mathrm{~cm}$ de 


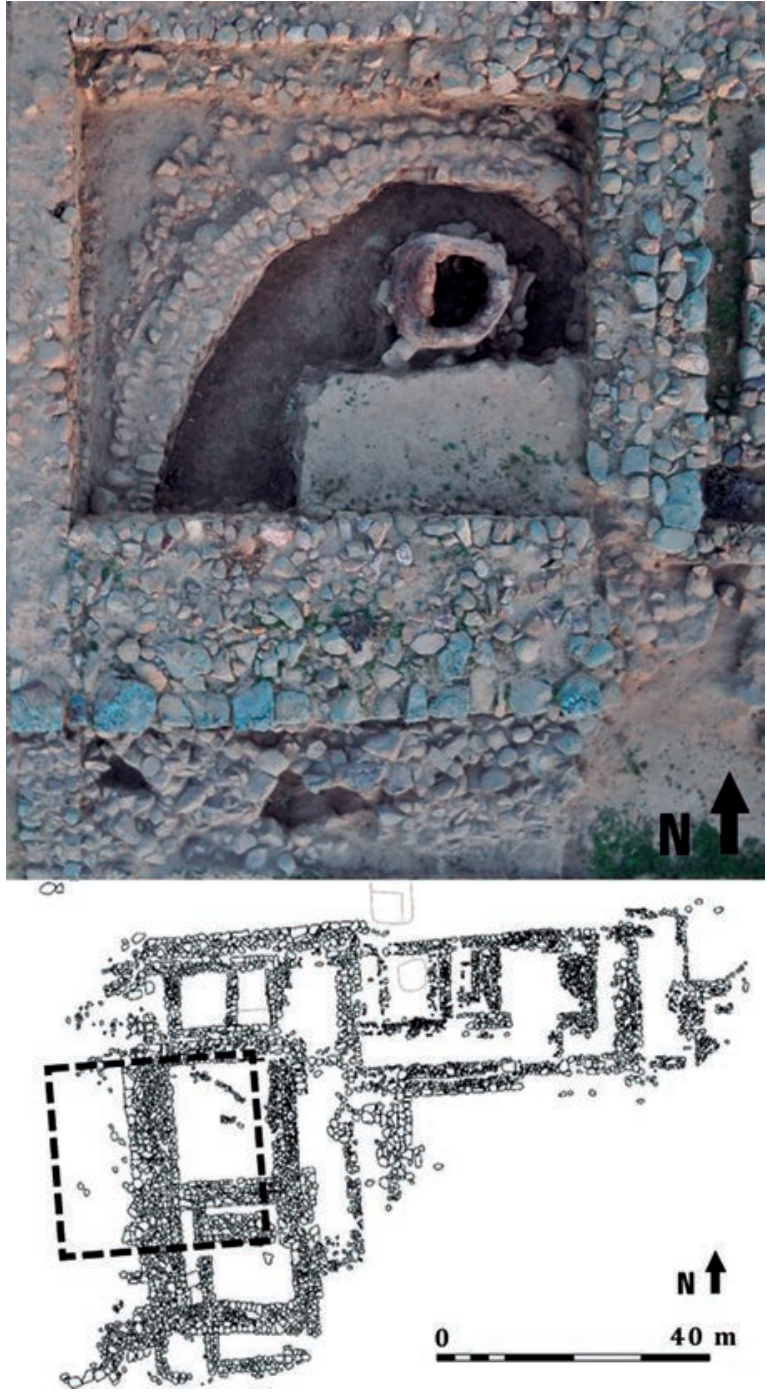

Fig. 8. Vista aérea de la cabaña oval de la Fase III de Cerro Borreguero (Zalamea de la Serena, Badajoz) (en color en la edición electrónica). histórico, el interior de la construcción oval fue amortizado con cantos de río de gran tamaño dispuestos en seco. Así mismo, la cabaña disponía de un hogar circular en su lado oriental que no fue destruido, sino que sobre su estructura se levantaron los hogares de las fases protohistóricas; es decir, se mantuvo el mismo eje en las diferentes fases de ocupación del lugar, lo que da una idea de la importancia simbólica del sitio.

La cabaña oval ha sido fechada en el siglo IX ane a partir de un análisis de C14 (Fig. 9) realizado en unos restos de carbón de madera localizados en su interior. La muestra, calibrada al 95\% aporta una cronología de Beta-406622: $2740 \pm 30 \mathrm{BP}$, Cal BC 970 to $960 / \mathrm{Cal}$ $\mathrm{BC} 930$ to 820 ; por lo que fijamos la cronología de su uso en el siglo IX ane y su abandono y amortización a finales del siglo VIII ane, momento en el que parece que convive con la construcción cuadrangular de esquinas redondeadas de la fase IIb. Esta cronología casa con el material documentado en la excavación de la estructura, que si bien es escaso, está fabricado en su totalidad a mano, destacando las piezas con decoración bruñida y escobillada. La existencia de esta cabaña, así como la presencia de esquinas redondeadas en otros puntos del enclave, hace albergar la posibilidad de que existiera un pequeño poblado de cabañas bajo las construcciones romanas y protohistóricas, una hipótesis que se podrá ir corroborando a medida que se avance en la excavación del lugar.

Debemos destacar que nos encontramos ante la cabaña oval más completa y mejor documentada del Bronce Final del valle Medio del Guadiana. Pero quizá lo más interesante es que por vez primera se documenta en esta zona y de una forma tan clara la evolución desde la cabaña circular a las primeras construcciones de muros rectos que, por otra parte, marca la transición entre el Bronce Final y el I Edad del Hierro. Esta evolución ya se había documentado en otros yacimientos del sur peninsular (Aguayo et al. 1986; Chaves y de la Bandera 1991; Izquierdo 1998) como se ha tratado

altura, mientras que la anchura media del muro oscila entre $60-70 \mathrm{~cm}$. La cimentación fue levantada a base de cantos rodados de mediano y pequeño tamaño trabados con una arcilla muy plástica y compacta de color marrón. Por último, la superficie de los cimientos, así como el interior de la cabaña, conservaban aún restos de arcilla anaranjada muy plástica procedente de su alzado de tapial, hoy prácticamente desaparecido.

La cabaña parece que fue amortizada a finales del siglo VIII ane para construir sobre ella el edificio de planta cuadrangular de época protohistórica. Su amortización se hizo respetando su trazado original y la totalidad de sus cimientos de piedra. Al igual que el resto de las estancias que conforman el edificio proto-

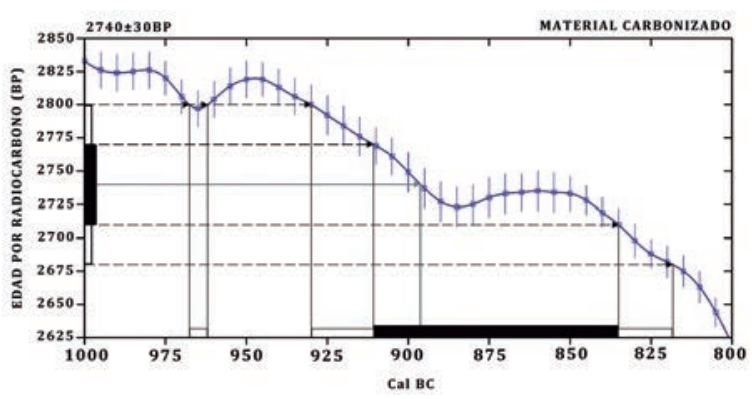

Fig. 9. Resultados de los análisis de $\mathrm{C} 14$ de muestras carbonizadas de madera de Cerro Borreguero (Zalamea de la Serena, Badajoz) (en color en la edición electrónica).

Trab. Prehist., 75, N. ${ }^{\circ}$ 1, enero-junio 2018, pp. 172-180, ISSN: 0082-5638

https://doi.org/10.3989/tp.2018.12211 
en un reciente trabajo (Celestino y Rodríguez González 2017), pero en el caso de Cerro Borreguero nos ilustra además sobre el marcado simbolismo y respeto por las construcciones antiguas que, por la forma en las que fueron amortizadas, debieron ostentar un marcado carácter público.

\section{UN YACIMIENTO DE TRANSICIÓN}

El yacimiento de Cerro Borreguero es el primer ejemplo con el que contamos para el estudio de la transición entre el Bronce Final y la I Edad del Hierro en el Guadiana Medio, una evolución que no se ha podido documentar fehacientemente en otros yacimientos de la zona por la ausencia de estructuras arquitectónicas que lo certifique, aunque no podemos olvidar que disponemos de otro ejemplo en el Bajo Guadiana ya mencionado en este trabajo, el caso de Castro dos Ratinhos, en la localidad portuguesa de Moura. De ese modo, el enclave aquí presentado tiene tres claras fases de ocupación que se corresponden, respectivamente, con el período romano, el tartésico y el Bronce Final (Fig. 10). Este último periodo está caracterizado por la construcción de una gran cabaña ovalada de aproximadamente $60 \mathrm{~m}^{2}$ de la que se conserva sus cimientos de piedra. Una vez amortizada la cabaña a finales del siglo VIII ane, se realizó un ritual en el que se utilizó al menos una cazuela a mano y pintada post-cocción cuyos fragmentos aparecieron dispersos sobre el cimiento de la cabaña. El primer edificio protohistórico se construyó hacia finales del siglo VIII ane, mientras que el segundo debió levantarse hacia mediados del siglo VII ane, abandonándose a comienzos del siglo VI ane tras limpiarlo y amortizarlo con piedras de mediano tamaño sin trabar.

El abandono del último edificio tartésico en torno a los primeros años del siglo VI ane coincide con el momento de construcción del primer edificio de Cancho Roano o "CR-C", lo que nos lleva a pensar que Cerro Borreguero sería el antecedente directo del santuario, construido a tan solo $2,5 \mathrm{~km}$ aguas arriba del río Ortiga. Carecemos de datos concluyentes que nos permitan saber cual fue la causa por la que se abandonó Cerro Borreguero; tal vez este cambio de estrategia se deba a que el río Ortigas se seca durante el período estival, al contrario de lo que sucede con el arroyo Cagancha que discurre al este del edificio de Cancho Roano y que se nutre durante todo el año de la vena de agua que pasa por debajo del mismo (Celestino 2005: 781); no obstante, es un argumento que está pendiente de poder ser contrastado con los pertinentes análisis paleoambientales del entorno. Igualmente, llama la atención cómo en el análisis del territorio en el que se insertan ambas construcciones se ha podido detectar que sendos edificios controlan vi-

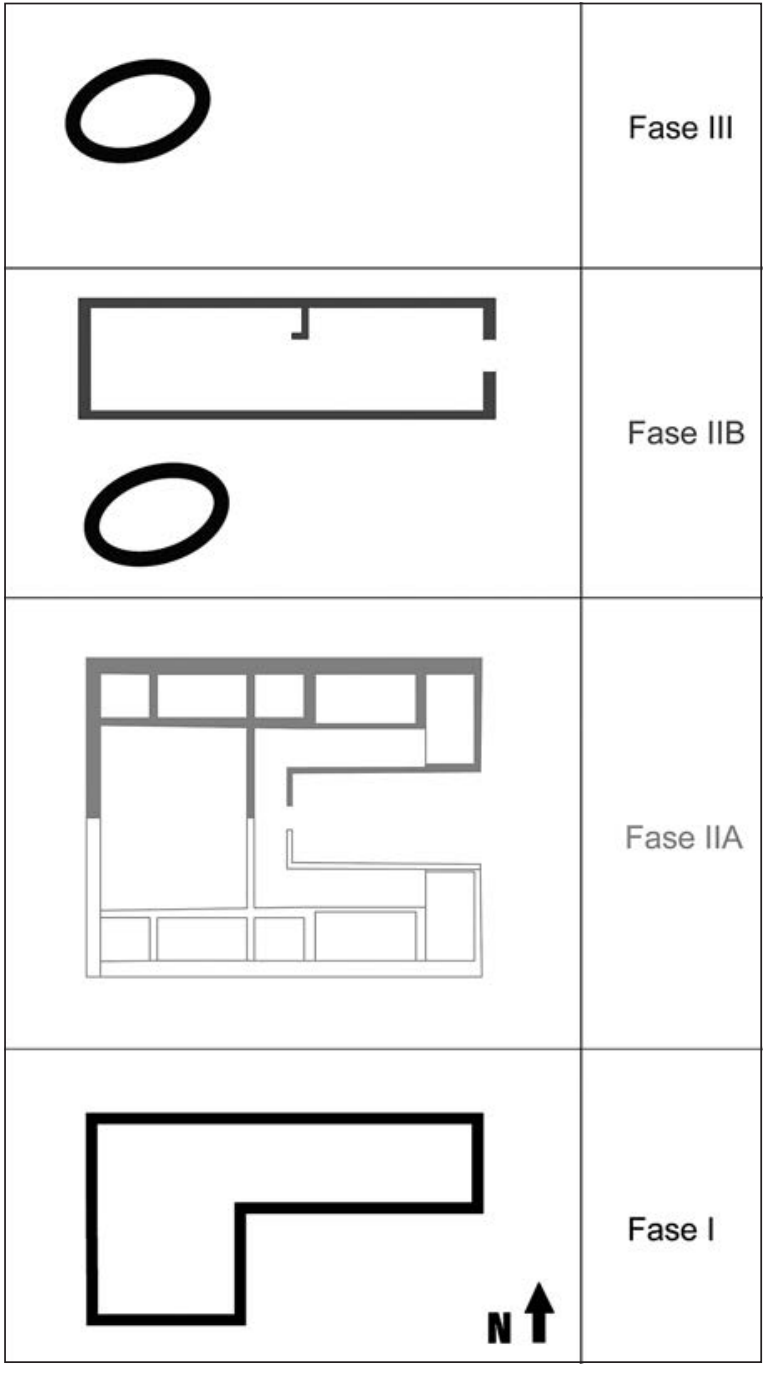

Fig. 10. Esquema de la evolución de las construcciones documentadas en las excavaciones de en 2008, 2010, 2012, 2013 y 2014 Cerro Borreguero (Zalamea de la Serena, Badajoz) (en color en la edición electrónica).

sualmente áreas distintas, sin que exista contacto alguno entre ellos; una cuestión que deberá ser analizada con detenimiento, pues otra de las razones del cambio podría radicar en la intención de hacer menos visible el enclave, ocultándolo en una vaguada dentro de la densa dehesa que lo rodea. En cualquier caso, las similitudes arquitectónicas que presenta el último edificio protohistórico de Cerro Borreguero con el primer santuario de Cancho Roano (Cancho Roano "C") son innegables, así como su contemporaneidad, lo que favorece la hipótesis sobre una continuidad constructiva entre ambos enclaves.

Por último, solo cabría otorgarle una funcionalidad a este edificio. Aunque parece una deducción lógica si 
aceptamos que Cerro Borreguero es el antecedente de Cancho Roano, resulta aún precipitado otorgarle una función netamente religiosa, como sin duda ostentó Cancho Roano en los tres edificios superpuestos que conocemos. Se han realizado prospecciones arqueológicas y geofísicas en el entorno inmediato de Cerro Borreguero para detectar estructuras de habitación que pudieran complementar la existencia de los edificios descritos, pero los resultados han sido fallidos, por lo que debemos considerar el enclave como un lugar aislado en el campo, en una ubicación y contexto muy similar al de Cancho Roano. Frente a ello, la presencia de grandes contenedores cerámicos y su proximidad a excelentes tierras de vegas dibujadas por el río Ortiga, lleva a pensar en el genuino carácter agrícola que tendría esta construcción, por otro lado obvia si se tiene en cuenta su localización en el llano. Pero como es habitual en este tipo de investigación, solo futuros trabajos podrán despejar las incógnitas que aún suscita el enclave, pero que sin embargo ya ha abierto una nueva vía para conocer la etapa de transición entre el Bronce Final y la I Edad del Hierro en la cuenca medida del Guadiana.

\section{AGRADECIMIENTOS}

Al Ayuntamiento de Zalamea de la Serena por la ayuda prestada en los trabajos de excavación, a Manuel Cumbre, propietario de la finca en la que se localiza el yacimiento de Cerro Borreguero, por permitirnos llevar a cabo los trabajos arqueológicos, así como al equipo de trabajo que durante estos años ha participado en las distintas campañas de excavaciones, especialmente, a José Ángel Salgado, codirector de la excavación durante las tres primeras campañas.

\section{BIBLIOGRAFÍA}

Aguayo, P.; Carrilero, M.; Flores, M. y Pino de la Torre, Mª 1986: "El yacimiento pre y protohistórico de Acinipo (Ronda, Málaga): un ejemplo de Cabañas del Bronce Final y su evolución". Arqueología Espacial 9: 33-58
Berrocal, L. y Silva, C. A. 2010: "O Castro dos Ratinhos (Barragem do Alqueva, Moura). Escavações num povoado proto-histórico do Guadiana. 2004-2007'. O Arqueólogo Português, suplemento 6.

Berrocal, L.; Silva, C. A. y Prados, F. 2012: "El Castro de Ratinhos, un ejemplo de orientalización entre las jefaturas del Bronce Final del Suroeste". En J. Jiménez Ávila (ed.): Sidereum Ana II. El río Guadiana en el Bronce Final. Anejos del Archivo Español de Arqueología LXII, CSIC-Mérida: 167-184.

Celestino, S. 2005: "El período Orientalizante en Extremadura y la colonización tartésica del interior". En S. Celestino Pérez y J. Jiménez Ávila (eds.): El Periodo Orientalizante. Actas del III Simposio Internacional de Arqueología de Mérida: Protohistoria del Mediterráneo Occidental. Anejos del Archivo Español de Arqueología XXXV, CSIC-Mérida: 227-235.

Celestino, S. y Cazorla, R. 2013: "La formación de un territorio tras la conquista romana: El caso de La Serena (Extremadura. España)". Estudios Romanos XVI: 171-190.

Celestino, S. y López-Ruiz, C. 2016: Tartessos and the Phoenicians in Iberia. Oxford University Press. Oxford.

Celestino, S. y Rodríguez González, E. 2017: "De lo invisible a lo visible. La transición entre el Bronce Final y la Primera Edad del Hierro en el valle medio del Guadiana". En S. Celestino y E. Rodríguez González (eds.): Territorios comparados los valles del Guadalquivir, el Guadiana y el Tajo en época tartésica. Anejos del Archivo Español de Arqueología LXXX, CSIC-Mérida: 183-212.

Chaves, F. y Bandera, M. L. de la 1991: "Aspectos de la urbanística en Andalucía occidental en los ss. VII-VI a.C. a la luz del yacimiento de Montemolín (Marchena, Sevilla)". Atti del II Congresso Internazionale di Studi Fenici e Punici (Roma 1987) II: 691-714. Roma.

Escacena, J. L. 2011: "Variación identitaria entre los orientales de Tartessos: reflexiones desde el antiesencialismo darwinista". En M. Álvarez Martí-Aguilar (ed.): Fenicios en Tartesos: nuevas perspectivas. British Archaeological Reports, International Series 2245, Archaeopress. Oxford: 161-192.

Esteban, C. y Escacena, J. L. 2013: “Arqueología del cielo. Orientaciones astronómicas en edificios protohistóricos del sur de la Península Ibérica". Trabajos de Prehistoria 70 (1): 114-139. https://doi.org/10.3989/tp.2013.12105

Fernández Flores, A. y Rodríguez Azogue, A. 2007: Tartessos desvelado. La colonización fenicia del suroeste peninsular y el origen y ocaso de Tartessos. Ed. Almuzara. Córdoba.

Izquierdo, R. 1998: "La cabaña circular en el mundo tartésico. Consideraciones sobre su uso como indicador étnico". Zephyrus 51: 277-288.

Prados, F. 2010: "La arquitectura sagrada: un santuario del siglo IX a.C.". En L. Berrocal Rangel y A. C. Silva (eds.): "O Castro dos Ratinhos (Barragem do Alqueva, Moura). Escavaçoes num povoado proto-histórico do Guadiana, 2004-2007'. O Arqueólogo Português, suplemento 6: 259-276.

Rodríguez González, E. y Celestino, S. 2017: "Las estancias de los dioses: la habitación 100 del yacimiento de Casas del Turuñuelo (Guareña, Badajoz)". Cuadernos de Prehistoria y Arqueología de la Universidad Autónoma de Madrid 43: 179-194.

Trab. Prehist., 75, N. ${ }^{\circ}$ 1, enero-junio 2018, pp. 172-180, ISSN: 0082-5638

https://doi.org/10.3989/tp.2018.12211 